\title{
Preeclampsia and Related Cardiovascular Risk: Common Genetic Background
}

\author{
Michalina Lisowska ${ }^{1}$ (D) Tadeusz Pietrucha $^{1} \cdot$ Agata Sakowicz $^{1}$
}

Published online: 3 July 2018

(C) The Author(s) 2018

\begin{abstract}
Purpose of Review Preeclampsia (PE) is a hypertensive disorder exclusive for pregnancy. It affects women all over the world and poses a great threat to life, both for mother and child. No definitive treatment exists and placenta delivery comprises the only known cure for PE. One of the most severe complications observed in preeclamptic women is the occurrence of cardiovascular diseases (CVDs) later in life.

Recent Findings Both PE and CVDs share some of their pathogenic pathways and gene variations. Thus far, a number of publications have examined those relationships; however, almost all of them focus only on common risk factors. The precise pathomechanism and genetic basis of PE and its associated cardiovascular complications remain unknown.

Summary Therefore, the aim of this review is to unify and clarify the current state of knowledge and provide direction for future studies, especially those regarding the genetic aspect.
\end{abstract}

Keywords Preeclampsia $\cdot$ Pre-eclampsia $\cdot$ Pregnancy $\cdot$ Cardiovascular disease $\cdot$ Cardiovascular risk $\cdot$ Genetics

\section{Introduction}

Hypertension and its related anomalies constitute one of the most frequent complications of pregnancy. A systematic analysis by the WHO published in 2014 places hypertensive disorders among the direct causes of perinatal death in women [1]. A stepwise reduction in maternal mortality has been observed over the last 25 years, falling almost $44 \%$ globally between 1990 and 2015 [2]. Nonetheless, despite the undertaken efforts, there is still a broad spectrum of life-threatening disorders which has not been managed. One such example is preeclampsia (PE), a clinical syndrome known for centuries, but remains incurable [3]. This hypertensive disease is associated with long-term vascular and metabolic alterations, influencing cardiovascular risk later in life. Regardless of their relationship

This study was supported by a grant from the Medical University of Lodz, Poland (no. 503/0-078-01/503-01-003).

This article is part of the Topical Collection on Preeclampsia

Michalina Lisowska

michalina.lisowska@gmail.com

1 Department of Medical Biotechnology, Medical University of Lodz, Zeligowskiego 7/9, Łódź, Poland with PE, cardiovascular diseases (CVDs) continue to be leading causes of global mortality $[1,4]$. It was estimated for the US population that almost $40 \%$ of women and more than half of men free from CVD at the age of 50 years will develop CVD in later life [5]. Globally, it is predicted that two of the most common CVDs, stroke and ischemic heart disease, will be the top two leading causes of death in 2030 [6].

Although the relationship between CVDs and PE has been studied many times, the majority of available articles focus on the risk factors common for both disorders. Thus far, a number of studies implying probable polymorphisms and gene candidates related to PE and CVDs have been published. Nonetheless, only a few of them are genome-wide studies or meta-analyses, assessing the influence of multiple samples and data sources. Therefore, the objective of this review is to gather recent data from the area of pathogenesis and the possible genetic changes underlying PE and its cardiovascular alterations to pave the way for future studies.

\section{The Association of Preeclampsia and Future Cardiovascular Risk}

Current literature thoroughly describes the connection between PE and related cardiovascular alterations, and 
numerous publications examine the common risk factors between them. Most list obesity, hypertension, hyperlipidemia, diabetes mellitus or physical inactivity as the main risk factors $[7,8,9 \cdot \bullet, 10,11]$. The general risk of the development of cardiovascular alterations in women with prior hypertensive disease of pregnancy varies according to population. Some of the population-specific risk variations are presented in Fig. 1 [12]. According to Ahmed et al., globally, the most frequent manifestation of vascular disease is stroke; the risk of its development is increased 14.5-fold after PE [95\% confidence interval (CI) 1.3-165.1]. In contrast, angina stands as the rarest complication, with a hazard ratio (HR) of 1.53 (95\% CI: 1.09-2.15) [13].

Some studies suggest that hypertensive pregnancy itself does not influence cardiovascular alterations, but some preexisting risk factors may in fact determine later cardiovascular risk [8]. A similar conclusion was reached by Bellamy et al. who suggests the presence of a possible pathological phenotype in women with recurrent PE, putting them at risk of hypertension and CVDs [9]. In contrast, Hromadnikova et al. propose that CVDs in later life may develop as a result of undergoing epigenetic changes related to complicated pregnancy [14•].

Despite the wealth of acquired data, some questions remain unanswered: Whether PE pathogenesis causes cardiovascular alterations, whether PE develops as a result of previously hidden cardiovascular abnormalities or whether the risk of both diseases is linked with factors existing before pregnancy. To clarify these points, it is necessary to learn more of the pathogenesis of the disease and its underlying genetics.

\section{Preeclampsia Pathophysiology: Overview}

$\mathrm{PE}$ is a complex disorder exclusive for pregnancy. The diagnosis criteria comprise recognition of the new onset of hypertension with coexisting proteinuria [15]. Because PE has a

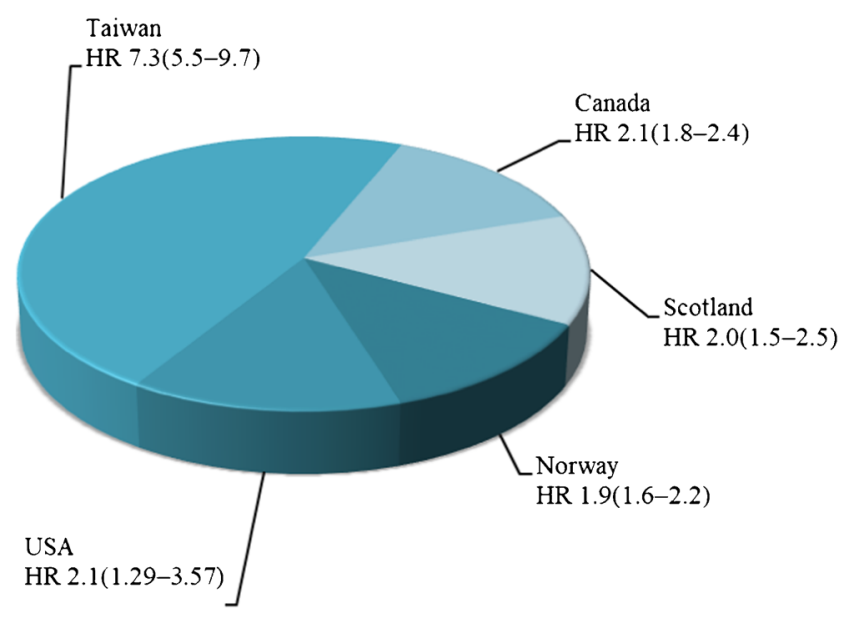

Fig. 1 Population-specific risk of cardiovascular disease or death in woman with pregnancy complicated by preeclampsia. HR- hazard ratio heterogeneous nature, its exact pathomechanism remains unclear; however, there are many theories addressing its behaviour. Most of them list oxidative stress, immunologic intolerance and angiogenic imbalance as the main causes [3, 16]. Despite the diversity of these theories, all share one common item, the placenta. The placenta is known to play a central role in PE pathogenesis as its presence is required for the development of the disease, and delivery of the placenta represents the only known cure [16].

The most important factors observed in the development of $\mathrm{PE}$ are inadequate trophoblast invasion and improper spiral artery development. In consequence, low-resistance vessels are not formed, leading to decreased blood flow and insufficient supply to the fetus. Eventually, these events trigger a restriction in uteroplacental circulation and result in an insufficiently perfused, ischemic and hypoxic placenta. As a consequence, local oxidative stress results in systemic inflammation, and the release of pro-inflammatory cytokines $[12,17]$. These changes cause endothelial dysfunction, which manifests itself as increased vascular permeability. Junction widening allows immune cells to infiltrate the vascular wall and exacerbate existing inflammation [18, 19]. Endothelial malfunctions are also observed in the kidneys: this specific glomerular lesion is known as 'glomerular endotheliosis' and it is associated with existing proteinuria [20].

Although abnormal utero-placental perfusion plays a significant role in the development of PE, some other maternal factors may significantly modulate the risk of this disease. Two of the most common subtypes of PE are known as early-onset PE and late-onset PE. Early-onset PE appears before the $34^{\text {th }}$ week of pregnancy and is usually manifested by low birth weight and intrauterine growth restriction (IUGR) and is believed to be of placental origin. In contrast, late-onset $\mathrm{PE}$ ( $\geq 34^{\text {th }}$ week) is generally related to maternal conditions such as obesity, diabetes or chronic kidney diseases [21-23]. The same clinical factors play a significant role in the development of CVDs [7, 8, 9••, 10, 11, 24].

Moreover, there is strong evidence that $\mathrm{PE}$ is connected with familial predispositions. A higher chance of PE has been found in the daughters of preeclamptic women during their own pregnancies [25]. In addition, a woman whose partner has previously fathered a preeclamptic pregnancy with another woman is also at higher risk of PE, implying that paternal genetic factors also play a role [26]. However, the inheritance effect along the maternal line is visibly stronger than the paternal one: 2.2 vs. 1.5 , respectively [27].

\section{The Association Between Preeclampsia Pathogenesis and Cardiovascular Disease}

Preexisting vascular endothelial dysfunction is currently viewed as a key common factor shared between PE and PE- 
related cardiovascular alterations. The elevated blood pressure typical for both medical conditions causes shear stress on the arterial wall, which promotes endothelial dysfunction and stiffness of the arteries. Moreover, dysfunction of the endothelium in the vessels is considered as an initial manifestation of arterial ageing [28]. A brief summary of such pathological changes is outlined in Fig. 2.

Inadequate spiral artery remodeling is one of the key characteristics of PE. During the development of PE, insufficient decidual arterial modification can result in their vasculopathy or acute atherosis. The former is characterized inter alia by

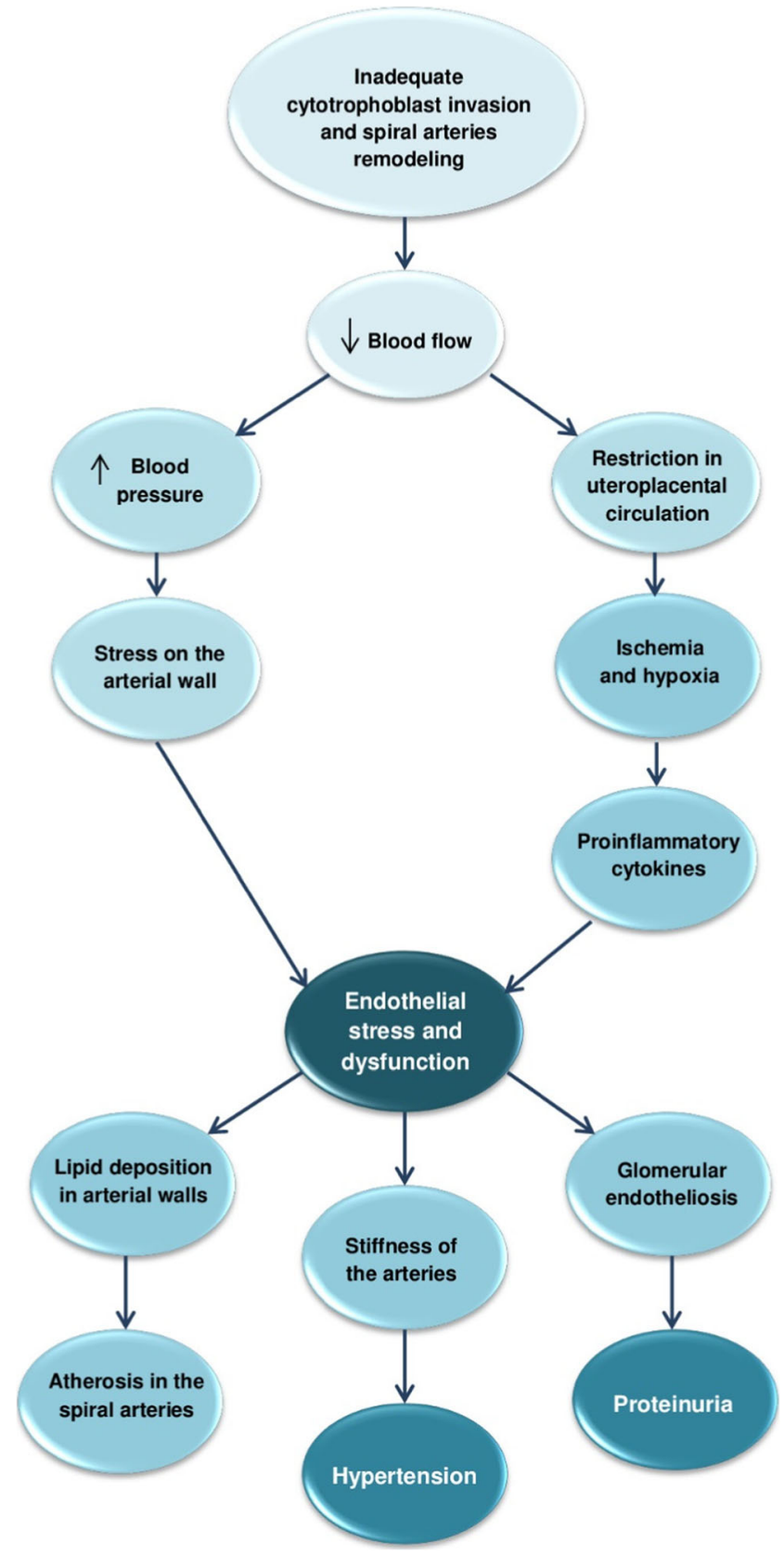

Fig. 2 Common pathogenic pathways for preeclampsia and preeclampsia-related cardiovascular alterations. Decrease $\downarrow$; increase $\uparrow$ foam cell infiltration into the vessel walls and subsequent constriction and high resistant flow. This results in elevated maternal diastolic blood pressure. Additionally, preeclamptic women diagnosed with decidual vasculopathy exhibit higher risk of chronic hypertension [29]. The latter, acute atherosis, develops solely in the spiral arteries. A possible explanation for its pathogenesis can be found in changes taking place in the uteroplacental circulation. Constricted, highly resistant vessels generate high flow velocity which may damage the villous surface architecture. Additionally, smooth muscle cells retained in improperly formed arteries escalate the hazard of spontaneous vasoconstriction and recurring perfusion of the intervillous space, generating ischemia- reperfusion injury $[30,31]$. As a consequence, two other characteristic components of both PE and CVDs are presented: inflammation and endothelial stress. The latter has been suggested to promote lipid deposition in the arterial walls, which may serve as an explanation for the development of acute atherosis in the decidual parts of the spiral arteries [30].

The generation of inflammation and endothelial stress results in enhanced production of pro-inflammatory factors. It was previously described that chronic inflammation may trigger foam cell formation [32]. Elevated levels of proinflammatory cytokines are responsible for monocyte and macrophage activation. Activated macrophages internalize lipoproteins, creating foam cells which together with formed fibrotic plaques are the key characteristics of atherosclerosis, one of the most serious cardiovascular complications [33]. Depending on the activation manner, macrophages can be divided into classically (M1) and alternatively activated (M2) groups; the M1 phenotype is regarded as pro-inflammatory, and M2 as anti-inflammatory. The imbalance in the M1to-M2 ratio may lead to plaque development and impaired inflammatory response [33, 34]. Macrophages also demonstrate distributional changes in PE. However, there is a significant difference in assumptions considering both conditions. It is suggested that atherosclerotic plaque formation includes mainly M2 macrophages, while PE pathogenesis is linked with decreased M2 level $[34,35]$. The results of in vitro studies suggest that the presence of activated macrophages may contribute to inadequate trophoblast invasion and improper vessel remodeling [36]. To better understand the role of changes in macrophage phenotype in this area, future investigations are needed.

During PE, placental ischemia induces the production and release of placental agents including angiogenic factors known to be implicated in the progression of endothelial and vascular dysfunction, and subsequent hypertension [37]. Antiangiogenic FMS-like tyrosine kinase 1 (sFlt-1) antagonizes two important angiogenic agents: vascular endothelial growth factor (VEGF) and placental growth factor (PlGF). sFlt-1 binds to them, thus preventing their interaction with receptors. In vivo studies conducted on pregnant rats demonstrated that 
sFlt-1 administration induces hypertension, proteinuria and glomerular endotheliosis in animals [38]. Furthermore, the sFlt-1 level was proven to be elevated in preeclamptic patients and to correlate positively with the disease severity [37,39].

Another widely studied anti-angiogenic factor is soluble endoglin (sEng). sEng and sFlt-1 are believed to be responsible for endothelial dysfunction in vitro. Moreover, like sFlt-1, sEng levels are known to increase with the disease severity [40]. These factors are also related to the risk of CVD. sFlt-1 is linked to cardiovascular remodeling and was demonstrated to be elevated in patients with heart failure. Additionally, it was suggested as a potential biomarker of cardiovascular risk [41]. Similar facts have been established for sEng, as it is considered as a reliable candidate for an indicator of hypertension, hypercholesterolemia, endothelial dysfunction and cardiovascular risk $[42,43]$.

\section{Underlying Genetics}

Most current studies on the relationship between PE and CVDs are focused on clinical similarities and common risk factors. Based on the changes that take place, and the possibility that increased risk for both disorders may be dependent on pre-pregnancy factors, current studies should address genetic aspects.

The vast majority of the tested genes and polymorphisms are directly related to the alterations observed in the pathological processes underlying both CVDs and PE. One of the largest of such studies was conducted to identify the common transcriptomic signatures involved in PE and CVDs [44]. A novel, bioinformatic meta-analysis investigating gene expression profiles from cardiovascular patient blood samples and placental tissue samples from preeclamptic women by Stiras et al. found that of 181 and 925 genes differentially expressed in CVD and PE, respectively; 22 genes were common for both disorders. Among them were those involved in oxidative stress, inflammation-mediated cytokines and chemokines, interleukin signaling or B-cell activation, all of which are found to take part in the complex pathogenesis of both diseases [12, 17, 45-48].

Some studies emphasize that both PE and CVDs share several lipid metabolism abnormalities. Johansson et al. performed a whole genome transcriptional profiling in decidual basalis tissue from Norwegian preeclamptic cases and controls and in lymphocytes from Mexican-American cases. They found that over 100 genes demonstrate various expression profiles among preeclamptic and control cases. Between those genes, only ACOX2 showed an inverse correlation with triglyceride levels and PE status [49]. Furthermore, they found that one single nucleotide polymorphism (SNP) of the ACOX2 gene (rs4681689) was associated $(p<0.05)$ with triglyceride levels.
ACOX2 is a peroxisomal enzyme physiologically responsible for one of the stages in the degradation of branched-chain fatty acids and bile acid. Down-regulation of its gene, and subsequent ACOX2 deficiency, results in fatty acid and bile acid accumulation [50]. Johansson et al. also found that ACOX2 level was inversely correlated with triglyceride levels, suggesting that down-regulated ACOX2 is associated with increased circulating triglyceride levels [49]. It was previously described that an abnormal lipid profile is associated with increased oxidative stress and endothelial dysfunction [51]. Additionally, high triglyceride levels are well-known CVD risk factors, and were proven to be elevated in preeclamptic patients, together with total serum free fatty acid level [52-55]. Therefore, it was suggested that ACOX2 may be a possible genetic risk factor linking PE and related CVDs [49].

Although changes in the gene expression level are known to be related to PE pathogenesis, some studies have also implicated gene structural changes. Groten et al. assessed five different polymorphisms (Tyr113His EPHX1, 894G/T and 4a4bVNTR eNOS, Met235Thr AGT and -401T/C ESR1) previously found to be associated with CVDs and impaired endothelial function [49]. Blood samples were taken from populations of Caucasian subjects $(\mathrm{N}=250)$ and black African women $(\mathrm{N}=220)$. Of all the tested polymorphisms, only epoxide hydrolase 1 (EPHX1) and variable nucleotide tandem repeats in intron 4 of the endothelial nitric oxide synthase (eNOSI4) were found to have a statistically significant relationship with the course of PE and its development [56].

Microsomal EPHX1 is an enzyme involved inter alia in the regulation of the oxidation status of xenobiotic-derived intermediates [57]. It has also been found to play a protective role against oxidative stress in an in vitro study assessing the influence of EPHX1 on embryo development in coculture [58]. Therefore, it is probable that altered variants of the enzyme disrupt its proper function, thus contributing to the development of PE and CVDs by oxidative stress-mediated and epoxide-related cytotoxic damage. Another significant polymorphism concerns eNOS, an enzyme involved in nitric oxide (NO) synthesis in the vascular endothelium. NO, an important relaxing factor, is implicated inter alia in regulating vascular tone, myocardial contractility and inhibiting platelet aggregation [59]. It was proven in vivo that the altered expression of eNOS, possibly caused by gene polymorphism, results in NO synthesis defects. Moreover, eNOS-deficient mice were shown to develop hypertension, hyperlipidemia and metabolic insulin resistance [60]. As those alterations, together with constricted vessels, are one of the main features of both PE and CVD, it seems plausible that eNOS genetic variants may be relevant to the disease outcome.

Groten et al., report that carriers of the EPHX1 polymorphism on exon 3 (Tyr113) demonstrated 3.6- fold increased risk of PE development (95\% CI: 1.366-8.750), and those carrying the 
eNOSI4 VNTR4a polymorphism demonstrated a 1.7- fold increased risk (95\% CI: 1.105-2.705) [56]. This supports previous research from Finland, in which the EPHX1 polymorphism on exon 3 was reported to be linked with $\mathrm{PE}(\mathrm{N}=248)[61]$; however, the odds ratio for PE associated with the Tyr113 polymorphism was 1.61 (95\% CI: $1.12-2.32)$; this difference may result from diverse patient populations. Studies conducted on black South African patients and Turkish women further indicate the significant influence of population diversity, as no significant alterations between groups were found in either study [62, 63].In addition, previous studies on the eNOSI4 polymorphism conducted in India and Iran fail to confirm any increased susceptibility of 4 a allele carriers to PE [64, 65]. Nonetheless, the presence of differences in obtained outcomes may again suggest an interdependence between the polymorphism and type of population.

Another search conducted in the area was based on previous findings confirming the PE susceptibility locus on chromosome 2q22[66, 67]. This family-based study (Australian family cohort) included testing four different SNPs risk variants on the following genes: lactase (LCT; rs2322659), low density lipoprotein receptor-related protein 1B (LRP1B; rs35821928), rho family GTPase 3 (RND3; rs115015150) and grancalcin (GCA; rs17783344). All of the tested SNPs were found to be associated with PE and known cardiovascular risk factors, such as weight, height, waist-to-hip ratio, blood glucose and triglycerides [68••]. These findings again indicate that CVDs and PE genetic mechanisms are shared, at least to a certain extent.

Systemic inflammation, with the coexisting release of proinflammatory molecules, was previously mentioned as a common characteristic for both $\mathrm{PE}$ and related cardiovascular alterations. The key factor regulating inflammatory response, and which is implicated in inflammation-associated diseases, is nuclear factor kappa beta (NFkB) [69]. Cytokine-dependent NFkB activation, followed by macrophage mobilization, results in further release of chemokines and cytokines. One of the main factors activating $\mathrm{NFkB}$, and thus the mentioned process, is inhibitory kappa B kinase beta (IKKB/IKK2). However, IKKB and NFkB, known to be implicated in observed systemic inflammation, may also act independently from each other. Chen et al. suggest a dual role of the molecules, both in prevention of the inflammation and in simultaneous increase in local injury [70]. However, the mode of IKKB function is still conflicting, as it may act through diverse targets.

It has already been established that NFkB plays a broad role in CVDs. It was proven in vivo that persistent NFkB activation in mice with heart failure promotes adverse remodeling, endoplasmic reticulum stress and apoptosis [71, 72]. Furthermore, IKK/NF- $\mathrm{BB}$ activation in cardiomyocytes was shown to induce excessive inflammatory response and myocyte atrophy, resulting in cardiomyopathy and heart failure [73]. NFkB also plays a prominent role in the pathogenesis of PE. Its placental expression was measured to be nearly 10 fold higher in preeclamptic pregnant women than in healthy pregnancies [74]. Among other things, NFkB possibly influences the pathogenesis of PE by inducing increased trophoblastic apoptosis [75].

As mentioned before, the key NFkB regulator IKKB may act independently of its target molecule. Thus, there is a possibility that its role in PE and CVD pathomechanisms might also be distinct. With this in mind, testing IKKB expression levels in affected patients seems to be an approach worth considering.

\section{Epigenetics}

Not only genetics, but also epigenetics, may yield new insights into the pathogenesis of PE and CVDs. Hromadnikova et al. conducted a retrospective cohort study based on Caucasian pregnant women $(\mathrm{N}=160)$ [14•]. The researchers focused on exploring the microRNA maternal profile in whole peripheral blood, regarding its previously established relationship with cardiovascular and cerebrovascular diseases. The tested groups included pregnancies complicated with gestational hypertension, intrauterine growth restriction (IUGR), PE and normal pregnancies as controls. The findings reveal miR-100-5p and miR-125b-5p to be deregulated in clinically established PE. Additionally, those microRNAs were also found to be deregulated in two other studied groups [14•]. Previous studies confirm a possible association of miR-100-5p with PE. In vivo studies on mice have shown that specific inhibition of miR-100-5p significantly increased expression of IL-6, a pro-inflammatory cytokine known to be elevated in PE women [76, 77]. IL-6 has also been found to play a key role in the pathophysiology of CVDs [45].

Another approach described by Oudejans et al. is based around the epigenetic changes associated with elevated risk of the disease. The study outcome gives a set of 12 differentially methylated regions (DMRs) which may be possibly related to the pathogenesis of CVDs and PE. Moreover, the authors suggest that DMRs identified in this way may serve as a foundation of the future cohort studies [78••].

\section{Future Directions and Conclusion}

PE and PE-related CVD are known to have common pathogenic pathways. Among the numerous pathological changes taking place in both disorders, the most important ones are hypertension, endothelial dysfunction, local inflammation and oxidative stress. The most probable hypothesis of the relationship between the diseases includes the risk factors present before pregnancy, as well as the contribution of later epigenetic changes. Most importantly, the presented review 
underlines the need for more significant studies based on larger study populations and supplemented by follow ups to allow a more thorough understanding of the pathogenesis of complex PE and PE-related CVDs. Furthermore, it is essential to create specific gene sets dedicated to clearly defined ethnic populations. A holistic, yet population-specific, approach is needed to eventually yield measurable data, translating into disease prevention.

Acknowledgements The authors would like to thank mgr. Edward Lowczowski for English correction.

\section{Compliance with Ethics Guidelines}

Conflict of Interest The authors declare no conflicts of interest relevant to this manuscript.

Human and Animal Rights and Informed Consent This article does not contain any studies with human or animal subjects performed by any of the authors.

Open Access This article is distributed under the terms of the Creative Commons Attribution 4.0 International License (http:// creativecommons.org/licenses/by/4.0/), which permits unrestricted use, distribution, and reproduction in any medium, provided you give appropriate credit to the original author(s) and the source, provide a link to the Creative Commons license, and indicate if changes were made.

\section{References}

Papers of particular interest, published recently, have been highlighted as:

- Of importance

•- Of major Importance

1. Say L, Chou D, Gemmill A, et al. Global causes of maternal death: A WHO systematic analysis. Lancet Glob Heal. 2014;2:323-33.

2. Alkema L, Chou D, Hogan D, et al. Global, regional, and national levels and trends in maternal mortality between 1990 and 2015, with scenario-based projections to 2030: A systematic analysis by the un Maternal Mortality Estimation Inter-Agency Group. Lancet. 2016;387:462-74.

3. Bell MJ. A Historical Overview of Preeclampsia- Eclampsia. J Obs Gynecol Neonatal Nurs. 2010;39:510-8.

4. Pagidipati NJ, Gaziano TA. Estimating deaths from cardiovascular disease: A review of global methodologies of mortality measurement. Circulation. 2013;127:749-56.

5. Hayman LL, Helden L, Chyun DA, et al. A Life Course Approach to Cardiovascular Disease Prevention. J Cardiovasc Nurs. 2011;26: S22-34.

6. Deaton C, Froelicher ES, Wu LH, et al. The Global Burden of Cardiovascular Disease. Eur J Cardiovasc Nurs. 2011;10:S5-S13.

7. Valdiviezo C, Garovic VD, Ouyang P. Preeclampsia and hypertensive disease in pregnancy: Their contributions to cardiovascular risk. Clin Cardiol. 2012;35:160-5.

8. Romundstad PR, Magnussen EB, Smith GD, et al. Hypertension in pregnancy and later cardiovascular risk: Common antecedents? Circulation. 2010;122:579-84.
9.• Bellamy L, Casas J-P, Hingorani AD, et al. Pre-eclampsia and risk of cardiovascular disease and cancer in later life: systematic review and meta-analysis. Bmj. 2007;335:974. This is a metaanalysis including prospective and retrospective cohort studies based on 3,488,160 women. A total of 198,252 was affected by pre-eclampsia while 29,495 had episodes of cardiovascular disease and cancer

10. Riise HKR, Sulo G, Tell GS, et al. Incident coronary heart disease after Preeclampsia: Role of reduced fetal growth, preterm delivery, and parity. J Am Heart Assoc; 6. https://doi.org/ 10.1161/JAHA.116.004158.

11. Andersson P, Sjoberg R, Ohrvik J, et al. Knowledge about cardiovascular risk factors among obese individuals. Eur J Cardiovasc Nurs. 2006;5:275-9.

12. Chen CW, Jaffe IZ, Karumanchi SA. Pre-eclampsia and cardiovascular disease. Cardiovasc Res. 2014;101:579-86.

13. Ahmed R, Dunford J, Mehran R, et al. Pre-eclampsia and future cardiovascular risk among women: A review. J Am Coll Cardiol. 2014;63:1815-22.

14. Hromadnikova I, Kotlabova K, Hympanova L, et al. Cardiovascular and cerebrovascular disease associated microRNAS are dysregulated in placental tissues affected with gestational hypertension, preeclampsia and intrauterine growth restriction. PLoS One. 2015;10:1-28. This study tested 32 microRNA gene expression profiles and found that two of them, miR-100-5p and miR-125b-5p, are especially engaged in preeclampsia development

15. Diagnosis ACOG. management of preeclampsia and eclampsia. Int J Gynecol Obstet. 2002;77:67-75.

16. Powe CE, Levine RJ, Karumanchi SA. Preeclampsia, a disease of the maternal endothelium: The role of antiangiogenic factors and implications for later cardiovascular disease. Circulation. 2011;123:2856-69.

17. Valenzuela FJ, Pérez-Sepúlveda A, Torres MJ, et al. Pathogenesis of preeclampsia: The genetic component. J Pregnancy. 2012; https://doi.org/10.1155/2012/632732.

18. LaMarca B. Endothelial dysfunction. An important mediator in the pathophysiology of hypertension during pre-eclampsia. Minerva Ginecol. 2012;64:309-20.

19. Roberts JM, Taylor RN, Goldfien A. Clinical and BiochemicalEvidence of Endothelial-Cell Dysfunction in the Pregnancy Syndrome Preeclampsia. Am J Hypertens. 1991;4:700-8.

20. Manuscript A. NIH Public Access. Growth (Lakeland). 2008;23:1-7.

21. Ogge G, Chaiworapongsa T, Romero R, et al. Placental lesions associated with maternal underperfusion are more frequent in early-onset than in late-onset preeclampsia. J Perinat Med. 2011;39:641-52.

22. White WM, Mielke MM, Araoz PA, et al. A history of preeclampsia is associated with a risk for coronary artery calcification 3 decades later. Am J Obstet Gynecol. 2016;214:519.e1-8.

23. Kovo M, Schreiber L, Ben-Haroush A, et al. The placental component in early-onset and late-onset preeclampsia in relation to fetal growth restriction. Prenat Diagn. 2012;32:632-7.

24. Cohen DL, Townsend RR. Hypertension and Kidney Disease: What Do the Data Really Show? Curr Hypertens Rep. 2012;14:462-7.

25. Tálosi G, Endreffy E, Túri S, et al. Molecular and Genetic Aspects of Preeclampsia: State of the Art. Mol Genet Metab. 2000;71:565-72.

26. Lie RT, Rasmussen S, Brunborg H, et al. Fetal and maternal contributions to risk of pre-eclampsia: population based study, https:// www.ncbi.nlm.nih.gov/pmc/articles/PMC28531/pdf/1343.pdf (accessed 29 May 2018).

27. Skjaerven R, Vatten LJ, Wilcox AJ, et al. Recurrence of preeclampsia across generations: exploring fetal and maternal genetic components in a population based cohort. https://doi.org/10.1136/ bmj.38555.462685.8F. 
28. Elias-Smale SE, Günal A, Maas AHEM. Gynecardiology: Distinct patterns of ischemic heart disease in middle-aged women. Maturitas. 2015;81:348-52.

29. Stevens DU, Smits MP, Bulten J, et al. Prevalence of hypertensive disorders in women after preeclamptic pregnancy associated with decidual vasculopathy. Hypertens Pregnancy. 2015;00:1-10.

30. Staff AC, Dechend R, Redman CWG. Review: Preeclampsia, acute atherosis of the spiral arteries and future cardiovascular disease: Two new hypotheses. Placenta. 2013;34:S73-8.

31. Burton GJ, Woods AW, Jauniaux E, et al. Rheological and Physiological Consequences of Conversion of the Maternal Spiral Arteries for Uteroplacental Blood Flow during Human Pregnancy. Placenta. 2009;30:473-82.

32. Angelovich TA, Hearps AC, Jaworowski A. Inflammation-induced foam cell formation in chronic inflammatory disease. Immunol Cell Biol. 2015;93:683-93.

33. McLaren JE, Michael DR, Ashlin TG, et al. Cytokines, macrophage lipid metabolism and foam cells: Implications for cardiovascular disease therapy. Prog Lipid Res. 2011;50:331-47.

34. Fong CHY, Bebien M, Didierlaurent A, et al. An antiinflammatory role for IKK $\beta$ through the inhibition of "classical" macrophage activation. J Exp Med. 2008;205:1269-76.

35. Oh J, Riek AE, Weng S, et al. Endoplasmic reticulum stress controls M2 macrophage differentiation and foam cell formation. $J$ Biol Chem. 2012;287:11629-41.

36. Renaud SJ, Postovit L-M, Macdonald-Goodfellow SK, et al. Activated macrophages inhibit human cytotrophoblast invasiveness in vitro. Biol Reprod. 2005;73:237-43.

37. Hariharan N, Shoemaker A, Wagner S. Pathophysiology of hypertension in preeclampsia. Microvasc Res. 2017;109:34-7.

38. Maynard SE, Min J, Merchan J, et al. Excess Placental Soluble fms-like Hypertension, and Proteinuria in. $J$ Clin Invest. 2003;111:649-58.

39. Tang P, Xu J, Xie B, et al. Use of serum and urinary soluble sFlt-1 and PLGF in the diagnosis of preeclampsia. Hypertens Pregnancy. 2017;36:48-52.

40. Venkatesha S, Toporsian M, Lam C, et al. Soluble endoglin contributes to the pathogenesis of preeclampsia. Nat Med. 2006;12:642-9.

41. Gruson D, Hermans MP, Ferracin B, et al. Sflt-1 in heart failure: relation with disease severity and biomarkers. Scand J Clin Lab Invest. 2016;76:411-6.

42. Blázquez-Medela AM, García-Ortiz L, Gómez-Marcos MA, et al. Increased plasma soluble endoglin levels as an indicator of cardiovascular alterations in hypertensive and diabetic patients. $B M C$ Med. 2010;8:86.

43. Rathouska J, Jezkova K, Nemeckova I, et al. Soluble endoglin, hypercholesterolemia and endothelial dysfunction. Atherosclerosis. 2015;243:383-8

44. Sitras V, Fenton C, Acharya G. Gene expression profile in cardiovascular disease and preeclampsia: A meta-analysis of the transcriptome based on raw data from human studies deposited in Gene Expression Omnibus. Placenta. 2015;36:170-8.

45. Kanda T, Takahashi T. Interleukin-6 and Cardiovascular Diseases. Jpn Heart J. 2004;45:183-93.

46. Cordero-Reyes AM, Youker KA, Torre-Amione G. The role of Bcells in heart failure. Methodist Debakey Cardiovase J. 2013;9:15-9.

47. Fettke F, Schumacher A, Costa S-D, et al. B Cells: The Old New Players in Reproductive Immunology. Front Immunol; 5. 2014. https://doi.org/10.3389/fimmu.2014.00285.

48. Lockwood CJ, Yen C-F, Basar M, et al. Preeclampsia-related inflammatory cytokines regulate interleukin-6 expression in human decidual cells. Am J Pathol. 2008;172:1571-9.

49. Johansson A, Curran JE, Johnson MP, et al. Identification of ACOX2 as a shared genetic risk factor for preeclampsia and cardiovascular disease. Eur J Hum Genet. 2011;19:796-800.
50. Baumgart E, Vanhooren JCT, Fransen M, et al. Molecular characterization of the human peroxisomal branched- chain acyl-CoA oxidase: cDNA cloning, chromosomal assignment, tissue distribution, and evidence for the absence of the protein in Zellweger syndrome. Biochemistry. 1996;93:13748-53.

51. Sima AV, Stancu CS, Simionescu M. Vascular endothelium in atherosclerosis. Cell Tissue Res. 2009;335:191-203.

52. Siddiqui I. Maternal Serum Lipids in Women with Pre-eclampsia. Ann Med Health Sci Res. 2014;4:638-41.

53. Ray JG, Diamond P, Singh G, et al. Brief overview of maternal triglycerides as a risk factor for pre-eclampsia. BJOG An Int J Obstet Gynaecol. 2006;113:379-86.

54. Harchaoui K, Visser M, Kastelein J, et al. Triglycerides and Cardiovascular Risk. Curr Cardiol Rev. 2009;5:216-22.

55. Villa PM, Laivuori H, Kajantie E, et al. Free fatty acid profiles in preeclampsia. Prostaglandins Leukot Essent Fat Acids. 2009;81:17-21.

56. Groten T, Schleussner E, Lehmann T, et al. ENOSI4 and EPHX1 polymorphisms affect maternal susceptibility to preeclampsia: Analysis of five polymorphisms predisposing to cardiovascular disease in 279 Caucasian and 241 African women. Arch Gynecol Obstet. 2014;289:581-93.

57. Farin FM, Janssen P, Quigley S, et al. Genetic polymorphisms of microsomal and soluble epoxide hydrolase and the risk of Parkinson's disease. Pharmacogenetics. 2001;11:703-8.

58. Cheong AWY, Lee Y-L, Liu W-M, et al. Oviductal microsomal epoxide hydrolase (EPHX1) reduces reactive oxygen species (ROS) level and enhances preimplantation mouse embryo development. Biol Reprod. 2009;81:126-32.

59. Loscalzo J, Welch G. Nitric oxide and its role in the cardiovascular system. Prog Cardiovasc Dis. 1995;38:87-104.

60. Duplain H, Burcelin R, Sartori C, et al. Insulin Resistance, Hyperlipidemia, and Hypertension in Mice Lacking Endothelial Nitric Oxide SynthaseNote Added in Proof. Circulation. 2001;104:342-5.

61. Laasanen J, Romppanen E-L, Hiltunen M, et al. Two exonic single nucleotide polymorphisms in the microsomal epoxide hydrolase gene are jointly associated with preeclampsia. Eur J Hum Genet. 2002;10:569-73.

62. Gebhardt GS, Peters WHM, Hillermann R, et al. Maternal and fetal single nucleotide polymorphisms in the epoxide hydrolase and gluthatione S-transferase P1 genes are not associated with preeclampsia in the Coloured population of the Western Cape, South Africa. J Obstet Gynaecol. 2004:24:866-72.

63. Pinarbasi E, Percin FE, Yilmaz M, et al. Association of microsomal epoxide hydrolase gene polymorphism and pre-eclampsia in Turkish women. J Obstet Gynaecol Res. 2007;33:32-7.

64. Aggarwal PK, Jain V, Jha V. Endothelial nitric oxide synthase, angiotensin-converting enzyme and angiotensinogen gene polymorphisms in hypertensive disorders of pregnancy. Hypertens Res. 2010;33:473-7.

65. Rahimi Z, Aghaei A, Rahimi Z, et al. Endothelial nitric oxide synthase (eNOS) $4 \mathrm{a} / \mathrm{b}$ and G894T polymorphisms and susceptibility to preeclampsia. J Reprod Infertil. 2013;14:184-9.

66. Moses EK, Lade JA, Guo G, et al. A Genome Scan in Families from Australia and New Zealand Confirms the Presence of a Maternal Susceptibility Locus for Pre-Eclampsia, on Chromosome 2. Am J Hum Genet. 2000;67:1581-5.

67. Johnson MP, Fitzpatrick E, Dyer TD, et al. Identification of two novel quantitative trait loci for pre-eclampsia susceptibility on chromosomes $5 \mathrm{q}$ and $13 \mathrm{q}$ using a variance components-based linkage approach. Mol Hum Reprod. 2007;13:61-7.

68.• Løset M, Johnson MP, Melton PE, et al. Preeclampsia and cardiovascular disease share genetic risk factors on chromosome $2 \mathrm{q} 22$. Pregnancy Hypertens. 2014;4:178-85. Study investigated four different single nucleotide polymorphism (SNPs) risk variants 
on the following genes: lactase, low-density lipoprotein receptor-related protein $1 \mathrm{~B}$, rho family GTPase 3 and grancalcin. All of the tested SNPs were found to be associated with preeclampsia and known cardiovascular risk factors, suggesting that mentioned diseases have the same genetic background

69. Baker RG, Hayden MS, Ghosh S. NF-kB, inflammation, and metabolic disease. Cell Metab. 2011;13:11-22.

70. Chen L-W, Egan L, Li Z-W, et al. The two faces of IKK and NF-kB inhibition: prevention of systemic inflammation but increased local injury following intestinal ischemia-reperfusion. Nat Med. 2003;9:575-81.

71. van der Heiden K, Cuhlmann S, Luong LA, et al. Role of nuclear factor $\mathrm{kB}$ in cardiovascular health and disease. Clin Sci. 2010;118:593-605.

72. Hamid T, Guo SZ, Kingery JR, et al. Cardiomyocyte NF-kB p65 promotes adverse remodelling, apoptosis, and endoplasmic reticulum stress in heart failure. Cardiovasc Res. 2011;89:129-38.

73. Maier HJ, Schips TG, Wietelmann A, et al. Cardiomyocyte-specific I B kinase (IKK)/NF- B activation induces reversible inflammatory cardiomyopathy and heart failure. Proc Natl Acad Sci. 2012;109:11794-9.
74. Vaughan JE, Walsh SW. Activation of NF-kB in placentas of women with preeclampsia. Hypertens Pregnancy. 2012;31:243-51.

75. Aban M, Cinel L, Arslan M, et al. Expression of nuclear factorkappa B and placental apoptosis in pregnancies complicated with intrauterine growth restriction and preeclampsia: an immunohistochemical study. Tohoku J Exp Med. 2004;204:195-202.

76. Aungier SR, Ohmori H, Clinton M, et al. MicroRNA-100-5p indirectly modulates the expression of Il6, Ptgs $1 / 2$ and Tlr 4 mRNA in the mouse follicular dendritic cell-like cell line, FL-Y. Immunology. 2015;144:34-44.

77. Lamarca B, Brewer J, Wallace K. IL-6-induced pathophysiology during pre-eclampsia: potential therapeutic role for magnesium sulfate? Int $J$ Infereron Cytokine Mediat Res. 2011;1:59-64.

78.• Oudejans C, Poutsma A, Michel O, et al. Genome-wide identification of epigenetic hotspots potentially related to cardiovascular risk in adult women after a complicated pregnancy. PLoS One. 2016;11: $1-10$. Study focuses on epigenetic changes associated with elevated risk of $P E$ and CVDs. The author suggests that differentially methylated regions identified in the study may serve as a foundation of future cohort studies 